\title{
MEJORANDO LA EFECTIVIDAD INSTRUCCIONAL DEL TEXTO EDUCATIVO EN CIENCIAS: PRIMEROS RESULTADOS
}

\author{
SANJOSÉ LÓPEZ, V.', SOLAZ PORTOLÉS, J.J. ${ }^{2}$ y VIDAL-ABARCA GÁMEZ, E. ${ }^{3}$ \\ ${ }^{1}$ Departament de Didàctica de les Ciències Experimentals $\mathbf{j}$ Socials. Universitat de València. \\ ${ }^{2}$ IB de Llíria. València. \\ ${ }^{3}$ Departament de Psicologia Evolutiva i de l'Educació. Universitat de València.
}

\begin{abstract}
SUMMARY
In this paper we compile and analyze the textual variables taking part in comprehension and learning processes on scientific texts. Next we propose a new classification which can be applicated to instructional text. This proposal is evaluated in an experiment with different groups of students and several learning tasks. The first results are discussed.
\end{abstract}

\section{INTRODUCCIÓN}

Parece bien probado, según señalan diferentes autores (Meyer et al. 1988, Otero 1990, Otero y Campanario 1990 , Renner et al. 1990, Santelices 1990, Wandersee 1988, Wood y Wood 1988), que el libro de texto sigue utilizándose en ciencias como un recurso didáctico básico en países y situaciones educativas distintas, aunque de modos bien diferentes. Incluso el célebre filósofo e historiador de la ciencia T.S. Khun (1987, p. 204) se refiere en uno de sus ensayos al libro de texto como «la única fuente mediante la cual la mayoría de las personas entran en contacto con las ciencias físicas (...) Como atestiguan muchas autobiografías, incluso el investigador científico no siempre se halla libre de la imagen de libro de texto obtenida durante sus primeros contactos con la ciencia».

No nos debe extrañar este uso tan extendido del libro de texto si tomamos en consideración algunas de las importantes funciones que puede ejercer de apoyo al profesor en: la toma de decisiones curriculares, la planificación de estrategias de enseñanza, las explicaciones científicas y la promoción del cambio conceptual en los estudiantes (Ruth y Anderson 1986).

Así pues, dado el papel central del texto educativo en la instrucción en ciencias, es comprensible el considerable aumento de estudios en los últimos años para la mejora de su diseño y conteniđo, si bien, a pesar de esta voluntad declarada, no exista todavía un acuerdo tácito de cómo llevar a cabo esta mejora (Staver y Bay 1989). Sin embargo, en lo que sí parece existir un gran consenso es en la pobre calidad instruccional de los textos actuales con fines pedagógicos (Britton et al. 1989). Una muestra de ello, Ia tenemos en el trabajo realizado por Aguire de Cárcer (1983) en el Estado español y en el que determina los niveles de razonamiento en el sentido piagetiano de la exposición de los contenidos presentados en los libros de texto de Física y Química de segundo de BUP, y donde comprueba que son superiores a los que los alumnos son capaces de utilizar. Idénticas conclusiones obtienen en los Estados Unidos Staver y Bay (1989) para los textos elementales de ciencias.

Una de las formas en que se utiliza el libro de texto en ciencias es como fuente informativa mediante su lectura directa. Perfetti (1986) distingue dos niveles de comprensión en la lectura:

a) Nivel intraproposicional o microestructural, donde el lector recodifica, de manera habitualmente automática, la información de las proposiciones o unidades de significado del texto.

b) Nivel interproposicional o macroestructural, donde el lector integra conscientemente la informacion sumistrada por las distintas proposiciones del texto.

Obviamente, para llegar al nivel macroestructural se tiene que haber superado el microestructural, lo que significa, por ejemplo, que, si el discurso textual presenta gran complejidad léxico-sintáctica, el lector se queda en la formación de la microestructura y no accede a la 
macroestructura textual (García Madruga y Martín Cordero 1987).

En la actualidad existe un acuerdo bastante generalizado en afirmar que el proceso de compresión lectora es de tipo interactivo. Ello implica que el significado no es algo que está en el texto, sino que, el sujeto lector lo va construyendo progresivamente (Spiro 1982). En este proceso de construcción de significado son esenciales tanto los esquemas de conocimiento previo del sujeto (Anderson y Pearson 1984, Wilson y Anderson 1986, Otero 1990), como sus estrategias lectoras (Brown et al. 1983, Scardamalia y Bereiter 1984). Además, un tercer aspecto a considerar en la lectura proviene de los objetivos que se persiguen con ella, pues se ha constatado que las estrategias que se desarrollan son diferentes según sean aquéllos (Áaronson y Ferres 1984, Wandersee 1988).

\section{OBJETIVOS E HIPÓTESIS}

Los objetivos del presente trabajo se centraron en el texto de ciencias y su influencia en las tareas habituales de aprendizaje académico. Pretendíamos:

1) Recopilar todas aquellas variables o características textuales que, de acuerdo con la literatura, se revelaban decisivas en los procesos de recuerdo y comprensión de textos.

2) Clasificar todas estas variables textuales de un modo tal que permitiera una aplicación sencilla y racional sobre cualquier texto de ciencias, con el fin de mejorarlo.

3) Poner a prueba algunas de estas variables textuales en sujetos de diferente conocimiento previo y estrategias de lectura, y en distintas tareas de aprendizaje.

Nuestra hipótesis fue que una manipulación de variables textuales siguiendo las directrices expuestas en la bibliografía conduciría, en la generalidad de los sujetos, a resultados significativamente mejores en cualquier prueba que midiera la formación de la macroestructura textual (recuerdo libre, resolución de problemas, etc.).

\section{VARIABLES TEXTUALES QUE INCIDEN EN LA COMPRENSION LECTORA EN CIENCIAS: UNA CLASIFICACIÓN}

El interés en el estudio del texto educativo de ciencias desde el punto de vista psicológico-diđáctico es muy reciente, prácticamente se puede situar su comienzo en la década de los 70 . En las investigaciones efectuadas tomando como base dicho texto se han analizado, entre otras cuestiones, las siguientes:

a) La estructura y organización del contenido (DeeLucas y Larkin 1990, Golden et al. 1988, Mayer 1985, Meyer et al. 1988, Rossi 1990). b) Su exigencia cognitiva (Aguirre de Cárcer 1983, Staver y Bay 1989).

c) Su legibilidad (Williams y Yore 1985, Wood y Wood 1988).

d) Su estilo expositivo (Strube 1989a).

e) Las cuestiones insertadas (Leonard 1984, 1987, $\mathrm{Pe}$ dersen et al. 1988).

f) Su utilización para el cambio conceptual (Hynd y Alvermann 1986, Roth y Anderson 1986).

g) Los organizadores de avance y el conocimiento de prerrequisitos (Healy 1989).

h) El lenguaje figurativo: analogías, símiles y metáforas (Gilbert 1989).

i) Las variables metacognitivas (Otero 1990, Otero y Campanario 1990).

j) Las estrategias de estudio de los alumnos (FergusonHessler y de Jong 1990, Wandersee 1988).

k) El papel del conocimiento previo o esquemas previos del lector (Otero 1990, Santelices 1990).

No obstante, debemos también citar los estudios realizados sobre prosa expositiva, no necesariamente científica, que nos han servido de base para esta investigación. Así, Duffy y sus colaboradores (1989) fijan sus objetivos sobre la estructura y organización del contenido textual; Britton y sus colaboradores (1989) destacan la importancia de una buena señalización del texto (en tipos de letra, títulos, etc.) y de su simplicidad léxica y sintáctica; y Anderson y Armbruster (1984) proponen toda una estrategia que incluye la unidad, coherencia y estructura textual, más las características del lector, para determinar la "consideración» de los textos didácticos con el potencial lector.

En todos estos trabajos mencionados aparecen distintas variables textuales que afectan los procesos de comprension en el aprendizaje de las ciencias. El primer problema que se nos presenta es el de la clasificación de todo ese cúmulo de variables. El criterio más riguroso sería, sin duda, la ciasificación a partir de los procesos cognitivos implicados en la comprensión, almacenamiento y recuperación del material escrito. Sin embargo, tal modelo parece aún lejano pese a los avances de la psicología cognitiva en ese terreno (García Madruga y Martín Cordero 1987). Estos mismos autores organizan las variables textuales en dos grandes grupos: las que no modifican la estructura textual y las que sí to hacen. Vidal-Abarca (1990), por su parte, agrupa las variables textuales según tres propiedades ocaracterísticas: cohesión, coherencia y estructura.

Por nuestra parte, ofrecemos a continuación una clasificación que es, a nuestro juicio, de mayor aplicabilidad en la mejora del libro de texto de ciencias. Organiza las variables textuales bajo cuatro aspectos: contenido, estructura organizativa, cohesión y estructura superficial. 


\section{Contenido}

Los aspectos relativos al contenido hacen referencia a las proposiciones contenidas en el texto. Se trata de una dimensión textual que cobra vital importancia en los textos científicos, ya que la adquisición de conceptos no va a llenar un vacío de ignorancia sino a integrarse en un cuerpo organizado de ideas y concepciones previas. Por lo cual, se han de tener en cuenta en el contenido las preconcepciones de los alumnos (Carey 1986, Driver 1989, Driver et al. 1989, Hierrezuelo y Montero 1989), y en la introducción de conceptos se debe enlazar siempre con el conocimiento previo del alumno (Anderson y Armbruster 1984, Corral Iñigo et al. 1987, Driver 1988, Otero 1985, Roth y Anderson 1986, Wandersee 1989). Por otra parte, como señalan diferentes autores, no es menos importante la redundancia en la presentación y desarrollo de ideas claves o problemáticas (Mayer 1985, Merrill 1983,1987, Reigeluth y Stein 1983, Reigeluth 1987, Roth y Anderson 1986), y las explicaciones que relacionan la información textual con el mundo real (Corral Iñigo et al. 1987, Roth y Anderson 1986, Strube 1989b).

Hay evidencias empíricas de que el uso de lenguaje figurativo -analogías sobre todo- resulta muy positivo en la enseñanza de las ciencias (Dupin y Joshua 1989,1990). De hecho, desde hace algún tiempo ya se venía defendiendo su inclusión en el discurso textual (Sari y Reigeluth 1982, Reigeluth y Stein 1983, Reigeluth 1987, Strube 1989a) porque es de gran utilidad al estudiante para construir esquemas en tópicos desconocidos (Bean et al. 1985); esto es, actúa como organizador de avance de información promoviendo el aprendizaje y retención de conceptos del texto (Ausubel 1976). Sin embargo, se tienen que hacer dos puntualizaciones a este respecto: que se utilizará el lenguaje fígurativo sólo si sus referentes son bien conocidos por el lector (pues solamente conceptos familiares pueden servir como referentes para dicho lenguaje si su finalidad es enseñar conceptos no familiares) (Äderson y Armbruster 1984), y que su uso será selectivo, ya que puede provocar efectos no deseados, como por ejemplo, en la retención de conceptos y en Ia actitud de los alumnos (Gilbert 1989).

Otra consideración de gran importancia en el contenido de los textos educativos en ciencias experimentales es la presentación que en ellos se hace de modelos y teorías. Desde el punto de vista epistemológico, hemos de tener en cuenta que las teorías científicas tratan de modelos ideales que se supone que representan, de modo más o menos simbólico y con alguna aproximación, ciertos aspectos de los sistemas físicos, y jamás todos sus aspectos. Las teorías no «retratan» la realidad, sino que son construcciones realizadas con conceptos, hipótesis y relaciones lógicas esencialmente diferentes de sus correlatos (Bunge 1981,1985). En conclusión, los modelos son bocetos conceptuales de objetos cuya existencia se asume y que forman parte de, al menos, una teoría.

Desde el punto de vista pedagógico es importante destacar la perniciosa costumbre que se tiene en los libros de texto de ciencias de presentar modelos y teorías como datos brutos de la realidad (Corral Iñigo et al. 1987). Un análisis de los manuales franceses y escoceses revela interesantes detalles en este problema (Meheut et al. 1988). Así, en muchos de los manuales franceses, los modelos son presentados como objetos reales, ocultando las reglas de correspondencia que fundamentan su validez y sus aspectos teóricos. Dicha presentación resulta a todas luces contradictoria con una iniciación a lametodología científica, y se aproxima más a la ideología y al dogmatismo. En un gran número de manuales escoceses se adopta un punto de vista empirista, y se espera que, a partir de determinadas experiencias, el alumno llegue a construir por sí mismo un modelo. Es decir, en este caso se ocultan al alumno los aspectos teóricos de la observación y se le muestra ésta como punto de partida del conocimiento científico (Hodson 1988). En la misma línea, un análisis de los argumentos y explicaciones presentados en libros de tex to de ciencias físicas ingleses y australianos (Strube $1989 \mathrm{~b}$ ) señala la ausencia de pasajes en dichos textos que discutan la función de teorías, leyes y modelos en los procesos de indagación científica.

En estas condiciones, es fácil entender las ideas erróneas de los estudiantes acerca de la naturaleza de la ciencia, las teorías y la construcción de modelos (Gilbert 1991) y los fracasos que éstos tienen para unir los modelos a las situaciones reales del laboratorio o de la vida fuera del centro educativo, pues no llegan a hacerlos funcionar como construccines hipotéticas y heurísticas, sin o como dogmas definitivos y cerrados (Martinand 1983). Corno corolario de todo lo dicho, parece aconsejable en el contex to educativo, por una parte, distinguir claramente entre lo que es un modelo científico y lo que es una observación; y por otra, proporcionar las explicaciones oportunas sobre el concepto de modelo científico (Anderson 1990, Renström et al. 1990, LLorens 1988).

Para acabar este apartado, nos ocuparemos de una característica frecuente de la ciencia de los textos escolares, que es la de limitarse a «enunciados declarativos» sin referirse a problemas concretos y sin mostrar su carácter predictivo o sus límites de validez (Astolfi 1988), además de soslayar casi por completo el contexto de indagación que condujo a la formulación de conceptos, leyes y teorías (Stinner 1989). Esto mismo se ve confimado por el análisis de textos de ciencias fúsicas efectuado por Strube (1989b), que revela que la mayoría de los textos contienen un alto indice de proposiciones no justificadas (unjustified statements) que son utilizadas para informar al lector de resultados y un bajo índice de proposiciones indagativas (inquiry statements), donde el autor describe las dificultades conceptuales y experimentales que acompañaron el desarrrollo de los conceptos científicos. Justamente en este punto, Otero (1985, 1989) sostiene que una de las dificultades para el aprendizaje significativo de textos de ciencias radica en la ausencia de problemas que permitan disminuir la arbitrariedad aparente que tienen para el aprendiz.

\section{Estructura organizativa}

Dado que los macroprocesos lectores procuran una representación global del contenido del texto, es lógico que cuanto mayor sea la organización del texto más 
fácilmente se lleven a cabo dichos macroprocesos. Existen varios resultados de investigación que avalan esta conclusión (Brooks y Dansereau 1983, Danner 1976, Golden et al. 1988, Kintsch y Yarbrough 1982).

Como punto de partida para la organización de los textos se ha de procurar que dicha organización favorezca la activación de los esquemas de conocimiento necesarios para la recepción del nuevo material, ya que esto es crucial para la recuperación de la memoria del material significativo de cara a la comprensión. En este sentido, podemos citar el trabajo de Dee-Lucas y Larkin (1990), en el que se indican los beneficios de aquellos tipos de organización textual que subrayan los principios conceptuales situándolos al comienzo de los textos o párrafos, frente a otros tipos de organización que resalian las reglas formales y los hechos. También destacan los trabajos de Sari y Reigeluth (1982), Reigeluth y Stein (1983) y Reigeluth (1987), en los que se plantea una secuencia organizativa que se inicia con las ideas más simples, las mejor conocidas por el alumno, o las más familiares, para después elaborar cada una de ellas, y volver al final de la secuencia a sintetizar las ideas con un mayor grado de precisión, complejidad y abstracción.

Tambiên es necesario que en la estructura organizativa đel tex to haya una presentación adecuada de los tópicos que en él se tratan. Así, se ha observado la gran importancia de situar el tema o tópico del discurso al comienzo del tex to (Kieras 1978), y se recomienda darlos a conocer mediante títulos, encabezamientos o frases (Anderson y Armbruster 1984). Todas estas observaciones son acordes con las teorías de la comprensión que ponen énfasis en eI papel de la información supraordinada como gúa en el procesamiento del texto (Kintsch y van Dijk 1978, van Dijk y Kintsch 1983).

Un aspecto de la estructura organizativa, que pese a ser de público conocimiento no por ello suele ser menos descuidado, es el de utilizar párrafos distintos para unidades de información diferente (Mayer 1985). Ello aumenta la coherencia del discurso y demanda menos esfuerzo cognitivo por parte del alumno en este nivel de procesamiento (Craik y Lockhart 1972).

\section{Cohesión}

Puesto que ya conocemos algunos de los procedimientos para mejorar la organización general de un texto, hemos de planteamos la necesidad de que el lector procese el texto en un nivel adecuado para poder beneficiarse de dicha organización. Esto es, el discurso textual tiene que poseer una cohesión intraproposicional e interproposicional tal que permita una descripción coherente del contenido semántico (Kintsch y van Dijk 1978, van Dijk y Kintsch 1983). Así pues, la dimensión de cohesión hace referencia al conjunto de procedimientos que mantienen la conexión conceptual entre las diversas ideas que se encuentran en las frases y párrafos (Mayer 1985).

Para mejorar la cohesión intraproposicional o microestructural se recomienda especialmente reducir la com- plejidad léxica y sintáctica (Britton et al. 1989, Santelices 1990, Williams y Yore 1985), de este modo los aprendices recodifican sin dificultad las unidades de información a nivel microestructural y no sobrecargan la memoria (García Madruga y Martín Cordero 1987).

La variable complejidad léxica se puede reducir empleando vocabulariocientífico-técnico sólocuando sea estrictamente necesario por formar parte intrínseca del contenido y, en este caso, proveeremos al sujeto lector de definiciones claras (Anderson y Armbruster 1984). Otro problema del vocabulario en la enseñanza de las ciencias es el de la polisemia de los términos. En este sentido, se ha descubierto que existe una gran influencia de las acepciones cotidianas de dichos términos en la ađquisición de conceptos científicos (Llorens et al. 1989).

Se ha comprobado que una de las características del lenguaje de los libros de texto de ciencias es su proximidad al utilizađo por la literatura científica, donde aparecen oraciones estructuralmente complejas, con gran cantidad de palabras que el lector debe inferir, y de formas verbales en pasiva (Santelices 1990, Strube 1989a). Uno de los modos en que podemos paliar esta complejidad sintáctica es mediante la disminución del tamaño de la frase, que suele tener dos efectos: una mayor integración de ideas por su menor número, y evitar construcciones sintácticas menos usuales y más complejas como es el caso de la voz pasiva (Britton et al. 1982).

Como podemos intuir, la cohesión textual no queda solucionada concibiendo el texto como un simple conjunto de oraciones sintácticamente sencillas que exponen los contenidos en una secuencia más o menos lógica. Se ha de proporcionar una buena red informativa de manera que el aprendiz pueda establecer las oportunas interrelaciones entre conceptos (Duffy et al. 1989, Staver y Bay 1989). Es aquí, en consecuencia, donde desempeña un papel crucial la cohesión interproposicional o macroestructural. Vamos a señalar a continuación algunas técnicas para perfeccionarla:

1. Establecer explícitamente las relaciones entre ideas; a saber: el sujeto lector tiene que poder construir redes semánticas con un mínino de inferencias textuales. El uso de frases cortas y simples puede impedir la formación de estas redes (Anderson y Armbruster 1984), por tanto, se debe llegar a una situación de compromiso en el tamaño tal de frase que no impida la cohesión microestructural, pero que a su vez, favorezca la macroestructural.

2. Evitar las ideas irrelevantes para el contenido tratado. Cada idea presentada ha de contribuir a la integración de las proposiciones en la red semántica iniciada (Anderson y Armbruster 1984, Duffy et al. 1989). Se ha comprobado que, adicionando detalles de bajo nivel, se reduce el recuerdo de las ideas principales porque se distrae la atención del lector de la estructura central (Meyer 19 84).

3. Facilitar la unión entre los referentes y los referidos (o antecedentes) en el texto (Bernárdez 1982). A título de ejemplo, veamos un caso de desunión referente/referido: 
«La energía se utiliza para producir calor y electricidad. Por medio de ella funcionan las calefacciones, las máquinas y los motores.»

Existe un referente, el pronombre ella, que hace alusión a un referido citado con anterioridad, la energía. Sin embargo, se puede apreciar cómo, en este caso, podría haber confusión entre «energía» y «electricidad». Es decir, el texto hubiera tenido poca cohesión debido a la ambigüedad de la unión referente/referido. En cualquier caso, para que la unión referente/referido sea adecuada, se ha de evitar cualquier tipo de ambigüedad, así como que haya una gran distancia entre ambos, ya que en este caso se dificulta la comprensión tal y como predice eI modelo de comprensión de Kintsch y van Dijk (1978), debido a los procesos adicionales de búsqueda de información en la memoria a largo plazo que el lector tiene que realizar. Estos procedimientos de cohesión textual son particularmente importantes cuando los lectores son niños o adolescentes, a causa de su dificultad para inferir los contenidos y las relaciones ausentes (Anderson y Armbruster 1986).

4. Aumentar la conectividad entre frases. Esto se puede hacer mediante conjunciones, expresiones conjuntivas $\mathfrak{u}$ otras expresiones (Anderson y Armbruster 1986, Mayer 1985). Por ejemplo:

"Como ya hemos estudiado, las leyes de la Mecánica Clásica permiten conocer la velocidad y la posición de cualquier cuerpo macroscópico. Sin embargo, ello no es posible si nos estamos refiriendo al mundo microscópico, como es el electrón en el átomo.»

5. Utilizar partículas que permitan al lector dirigir su atención y le faciliten las inferencias a efectuar en la lectura (Corral Iñigo et al. 1987). Así, en el ejemplo anterior, la conectiva adversativa sin embargo cumple ambas funciones.

Una explicación psicológica, para dar cuenta del papel de la cohesión textual en la comprensión lectora, menciona la posibilidad de que la cohesión del discurso permita ta elaboración de una representación altamente integrada o interconectada en la memoria $y$, por ende, una recuperación más eficiente. Este punto puede ser ilustrado por varios modelos de memoria (Anderson y Bover 1973, Kintsch 1972, 1974, Rumelhartet al. 1972). En estos modelos, la información es representada en la memoria como una red interconectada de nudos encadenados, en la cual los nudos simbolizan conceptos y los eslabones entre nudos las relaciones entre conceptos. La representación construida a partir de un discurso cohesionado sería más eficiente para la recuperación, porque Las múltiples interconexiones entre conceptos incrementa tanto el número de nudos a los que se puede acceder como el número de vías potenciales para acceder a un determinado nudo.

\section{Estructura superficial}

Bajo este epígrafe incluímos un conjunto de procedimientos que no alteran la estructura del texto al que van dirigidos, y que sirven de estímulo o indicio para un procesamiento del texto por parte del lector que le conduzca a su macroestructura. Recogemos seguidamente algunos de los procedimientos más convenientes para la didáctica de las ciencias:

1. Destacar las ideas principales contenidas en el texto (Duffy et al. 1989, Meyer 1984, Rossi 1990).

2. Emplear frases introductorias que anuncien el contenido del texto (preview statements), así como frases-resumen (Britton et al. 1982).

3. Utilizar títulos y encabezamientos que suministren más información estructural y que estén colocados adecuadamente; de este modo se prepara al lector para adentrarse de un modo rápido en la idea principal (Anderson y Armbruster 1984, García Madruga y Martín Cordero 1987, Corral lnigo et a1. 1987).

4. Ajustar el estilo lingǘstico del texto al estuđiante. Normalmente el estilo de los tex tos escolares de ciencias es el derivado del modelo "correcto» de escribir literatura científica: rígido, ausente de vividez y de lenguaje figurativo, y que pone el acento en exceso en las argumentaciones lógicas, Ias definiciones y el razonamiento formal (Strube 1989a).

\section{Otras características}

Vamos a englobar aquí todo el conjunto de variables textuales que no forman parte sensu stricto de la prosa expositiva científica, y que, no obstante, constituyen una parte muy importante de los textos instruccionales, sin la cual sería imposible el aprendizaje en ciencias. A saber: las cuestiones o actividades y las figuras.

En cuanto al papel de las actividades o cuestiones, parece no haber ninguna duda acerca de su influjo positivo en la comprensión y retención del texto y, cómo señalan diferentes estudios, éste se debe a la focalización de la atención del aprendiz hacia determinadas partes del tex to (García Madruga y Martín Cordero 1987). Leonard $(1984,1987)$, por su parte, en sus trabajos sobre el efecto de las cuestiones insertadas en textos de ciencias, recoge abundante bibliografía que confirma la mayor productividad de las cuestiones cuando están colocadas después de Ios pasajes a los que se refieren, y la mayor eficacia de las cuestiones de alto nivel cognitivo (de comprensión, de inferencia y de aplicación) frente a las factuales. Este último resultado, junto con el obtenido por Pedersen y colaboradores (1988) que indica que los estudiantes que justifican las respuestas de las cuestiones recuerdan significativamente más el contenido textual, constatan el hecho de que cuantas más operaciones cognitivas se requieren al lector tanta más información recuerda posteriormente.

Por otro lado, las cuestiones son la clave de la utilización del texto instruccional como herramienta para el cambio conceptual cuando cumplen los siguientes requisitos: están centradas en las ideas principales, revelan errores 
conceptuales, promueven la unión entre las ideas del texto y la vidad real, sirven para poner en tela de juicio los preconceptos y dan muchas oportunidades de trabajar sobre un concepto (Roth y Anderson 1986).

Otra variable que es necesario considerar en los textos educativos de ciencias son las figuras, sobre todo en niveles elementales donde el aprendizaje de conceptos depende de la visualización de objetos y modelos icónicos (Storey Vasu y Howe 1989). Por tanto, parece inexcusable la presencia de ilustraciones en los textos, si bien no siempre mejoran la comprensibilidad de los mismos (Reynolds 1987). Los recientes resultados obtenidos por Mayer $(1989,1990)$ confirman que la efectividad de las ilustraciones en tareas de comprension y de recuerdo se halla ligada, entre otras cosas, a la explicación de la figura y a una buena conexión con el discurso textual. Con ello, el lector concentra sus esfuerzos sobre la información relevante y la organiza de un modo más coherente.

\section{METODOLOGÍA}

\section{Materiales}

Para nuestro estudio empleamos dos textos, que llamamos natural y adaptado. El texto natural empleado fue una copia literal de parte de la lección de un libro de texto de Física y Química de segundo de BUP con amplia difusión en el Estado español (Candel et al. 1988) correspondiente a Modelos atómicos; en concreto, la que comienza con las hipótesis atómicas de Datton hasta llegar al modelo de Rutherford y las partículas subatómicas: protón, neutrón y electrón. El texto constaba đe unas 1600 palabras y no incluía las actividades o cuestiones propuestas, pues nuestros objetivos se habían fijado preferentemente en la prosa expositiva y quisimos de este modo evitar sus posibles interferencias.

EI texto adaptado fue elaborado por nosotros a partir de la misma información básica proporcionada por el tex to natural (Solaz Portolés 1992). Estaba compuesto de alrededor de 1900 palabras, y en él incluímos algunas ideas no presentes en el texto natural ađemás de eliminar otras.

La introducción de nuevas ideas y la eliminación de otras en el tex to adaptado a partir del natural formaba parte del conjunto de cambios efectuados en el texto natural y plasmados en el adaptado. Los cambios comportaron la manipulación de un total de 23 variables textuales que, según la bibliografía consultada, suponían un perfeccionamiento en pos de conseguir un mayor aprendizaje, comprensión y retención del tex to (Solaz Portolés 1992). Además, de acuerdo con las directrices expuestas en la revisión teórica del apartado anterior, fueron clasificadas en cinco categorfas: contenido, estructura organizativa, cohesión, estructura superficial, y otras características.

La tabla I recoge todas esas variables.
Tabia I

\section{Contenido}

1. Tener en cuenta las preconcepciones del lector $\mathrm{e}$ introducir los conceptos a partir de su conocimiento previo.

2. Ser redundantes en la presentación y desarrollo de ideas importantes o problemáticas.

3. Proporcionar explicaciones que relacionen la informacion textual con el mundo real del lector.

4. Usar analogías.

5. Exponer el concepto de modelo científico y sus virtualidades.

6. Presentar el contenido conceptual como respuesta a problemas concretos y no como meros «enunciados declarativos».

\section{Estructura organizativa}

7. Subrayar los principios conceptuales situándolos al comienzo del texto, apartados o párrafos.

8. Partir de lo más conocido, sencillo o familiar.

9. Dar a conocer los distintos tópicos a tratar en títulos, encabezamientos of frases.

10. Utilizar párrafos distintos para unidacies de información diferente.

\section{Cohesión}

11. Reducir la complejidad léxica y sintáctica.

12. Establecer relaciones explícitas entre ideas de tal manera que se reduzcan las inferencias textuales.

13. Eliminar ideas irrelevantes para el contenido tratado.

14. Facilitar la unión entre referentes y referidos.

15. Aumentar la conectividad entre frases.

16. Emplear partfculas que dirijan la atención del lector y le faciliten las inferencias durante la lectura.

\section{Estructura superficial}

17. Destacar las ideas principales mediante tipos en negrita.

18. Utilizar frases introductorias que anuncien el contenido, así como frases resumen.

19. Ofrecer títulos y encabezamientos que suministren más información estructural y estén bien colocados.

20. Ajustar el estilo lingüístico al lector.

Otras características

21. Incluir figuras para incrementar la cohesión textual.

22. Explicar convenientemente las figuras e intercontectarlas con la prosa.

23. Simplificar y eliminar expresiones numéricas quc cran innecesariamente complejas.

\section{Muestras de lectores}

Participaron en nuestra investigación un total de 85 sujetos distribuidos en ocho grupos: UCN, UCA, ULN, ULA, BCN, BCA, BLN y BLA, donde U/B se refiere a universitarios/bachilleres, $C / L$ alude a ciencias/letras y N/A significa texto leído natural/adaptado.

La distribución de sujetos según el tipo de texto, natural o adaptado, fue totalmente aleatoria. La tabla adjunta (Tabla II) muestra el número y porcentaje de sujetos de cada grupo.

Tabla II

\begin{tabular}{|l|c|c|c|c|}
\cline { 2 - 5 } \multicolumn{1}{c|}{} & \multicolumn{2}{c|}{ Texto Natural } & \multicolumn{2}{c|}{ Texto Adaptado } \\
\cline { 2 - 5 } \multicolumn{1}{c|}{} & Universitarios & Bachilieres & Universitarios & Bachilleres \\
\hline Ciencias & 10 & 10 & 12 & 10 \\
& $(11.8)$ & $(11.8)$ & $(14.1)$ & $(11.8)$ \\
\hline Letras & 10 & 12 & 10 & 11 \\
& $(11.8)$ & $(14.1)$ & $(11.8)$ & $(12.8)$ \\
\hline
\end{tabular}


Los grupos de universitarios de ciencias (UC) estuvieron constituidos por alumnos de último curso de la licenciatura de Ciencias Físicas, especialidad de Física Teórica, de la Universitat de València, ya que se presuponía que éstos serían los de mayor conocimiento previo del tema sobre el que versaban los materiales de nuestro estudio: los modelos atómicos.

Los grupos de universitarios de letras (UL), los formaban estudiantes de quinto curso de la licenciatura de Psicologia, especialidad de Psicología Evolutiva y de la Educación, también de la Universitat de València. Fueron escogidos tanto por su presunto escaso conocimiento previo del texto que les presentaríamos, como por sus indudables buenas estrategias de lectura resultantes de su nivel académico y del tipo de estudios cursados.

Los grupos de bachilleres eran alumnos de tercero de BUP, de ciencias (BC) y de letras (BL), del Institut de Batxillerat de Quart de Poblet (Comarca de L'Horta Sud, València). Estos grupos fueron considerados pobres en estrategias de lectura en relación con los grupos de universitarios -no realizamos ninguna prueba para confirmarlo, pero los estudios de Brown y colaboradores (1983) y Kieras (1985) avalan esta suposición--, y con diferente conocimiento previo sobre modelos atómicos, presumiblemente.

El número de sujetos que participaron en la primera sesión fue de 109, y el de los que participaron en la segunda 85 (que son los que figuran en la tabla anterior). En consecuencia, hubo una mortandad experimental de 24 sujetos en la demora de una semana entre cada sesión.

\section{Procedimiento y medidas}

Obtuvimos tres tipos de medidas dependientes: dos que requerían la formación de la macroestructura textual, de recuerdo libre y de comprensión; y una que participa de manera muy importante en la ejecución de las dos anteriores, la captación de ideas principales. Para asegurarnos que la distribucion de los sujetos participantes se ajustaba a nuestras premisas, es decir, que los miembros de cada grupo tenían el conocimiento previo asignado, efectuamos con anterioridad una medida de conocimiento previo.

Las cuatro pruebas las llevamos a cabo en dos sesiones separadas por un período de una semana. La primera sesión constó de: la prueba de conocimiento previo (15 min.), lectura del tex to ( $25 \mathrm{~min}$.), y la prueba de recuerdo libre (20 min.). Entre la lectura del texto y la prueba de recuerdo libre medió un lapso de cinco minutos en tos que los sujetos hicieron tareas distractoras. La segunda sesión constó de la prueba de captación de ideas principales (25 min.) y de la de comprensión (20 min.).

La prueba de conocimiento previo se compuso de un cuestionario abierto de 6 ítems. Todos ellos versaron sobre electricidad porque consideramos estos conocimientos imprescindibles para la comprensión adecuada del tema de Modelos Atómicos. En la prueba de recuerdo, se pidió a los sujetos que escribieran todo lo que recordaran del texto que acababan de leer, sin ninguna restricción. Para la captación de ideas principales tuvieron el texto delante con unas instrucciones que leyeron previamente; en ellas se pedía que escribieran las siete u ocho ideas más relevantes del texto y que las formularan en forma de oración. La prueba de comprensión estuvo constituida por un cuestionario abierto de cinco ítems sobre Modelos Atómicos, tres de resolución de problemas (dos de ellos de tipo heurístico y uno de tipo algorítmico) y dos de inferencia textual; y también en esta prueba los sujetos dispusieron del texto.

En cada ítem de los cuestionarios de conocimiento previo y decomprensión se categorizaron las respuestas correctas y se puntuó la presencia/ausencia de las mismas (1/0). En la captación de ideas principales se calificaron las ideas presentes entre 0 y 2 puntos por comparación con las nueve ideas principales que los autores seleccionaron, según estuviera ausente, no completa o completa. Para la cuantificación de la medida de recuerdo libre, seguimos el modelo de cómputo de resultados de recuerdo propuesto por García Madruga y Martín Cordero(1987). La corrección de todas estas pruebas fue realizada por los autores, los cuales examinaron en común un tercio del total, obteniendo en todos los casos porcentajes de acuerdo superiores al $85 \%$ y resolviendo las discrepancias de común acuerdo.

\section{RESULTADOS}

Las puntliaciones medias (valores normalizados) en cada una de las pruebas pueden verse en las figuras adjuntas (Fig. 1, 2, 3 y 4 ).

\section{Figura 1}

Puntuaciones medias de la prueba de Conocimiento Previo Vatores normalizados: $0-1$

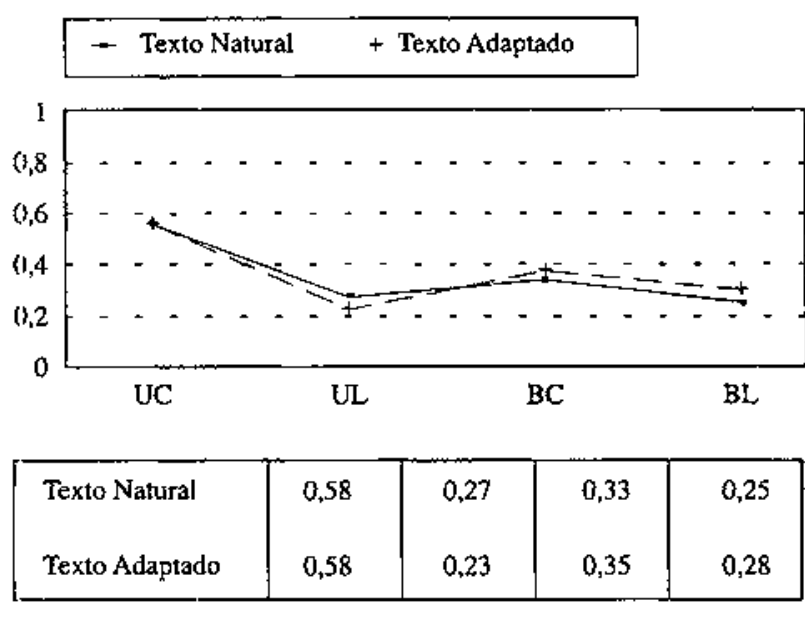

La mayor parte de los datos cuantitativos fueron analizados mediante un análisis de varianza con tres variables entre sujetos: conocimiento previo (con dos valores, 
Figura 2

Puntuaciones medias de la prueba de Recuerdo Libre Valores normalizados: 0 -I
Figura 4

Puntuaciones medias en la prueba de Comprensión Valores normalizados: $0-1$

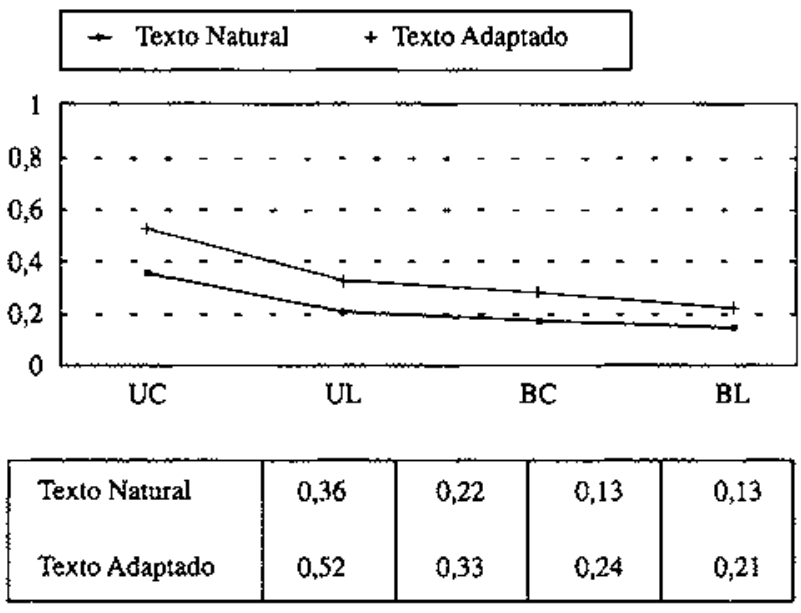

Figura 3

Puntuaciones medias en la prueba de Captación de Ideas Principales Valores normalizados: 0 - 1

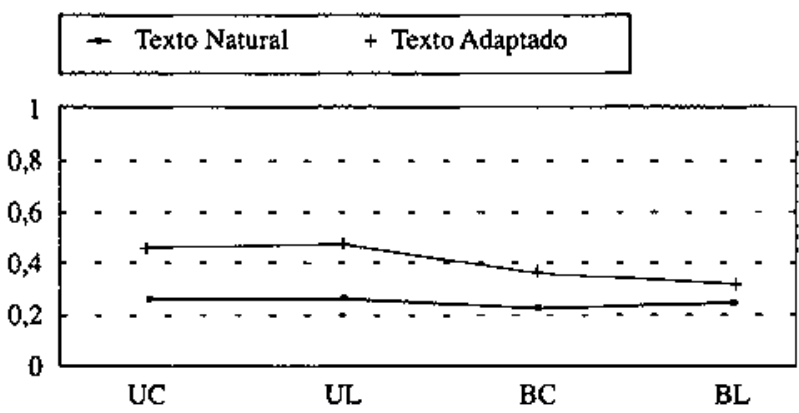

\begin{tabular}{|l|l|l|l|l|}
\hline Texto Natural & 0,26 & 0,26 & 0,22 & 0,24 \\
Texto Adaptado & 0,44 & 0,44 & 0,36 & 0,33 \\
\hline
\end{tabular}

ciencias y letras), estrategias de lectura (con dos valores, universitarios y bachilleres), y texto (con dos valores, natural y adaptado). Se tomaron como variables dependientes recuerdo libre, captación de ideas principales y comprensión, obteniéndose diferencias significativas en:

-Prueba de conocimiento previo: Estrategias de lectura, $\mathrm{F}(1,77)=22,3, \mathrm{p}<.001$, conocimiento previo, $\mathrm{F}(1,77)=$ $71,1, \mathrm{p}<.001, \mathrm{y}$ la interacción entre ambas variables, $\mathrm{F}(1,77)=27,2, \mathrm{p}<.001$.

-Prueba de recuerdo libre: Estrategias de lectura, $F(1,77)$ $=109,2, \mathrm{p}<.001$, conocimiento previo, $F(1,77)=44,6$,

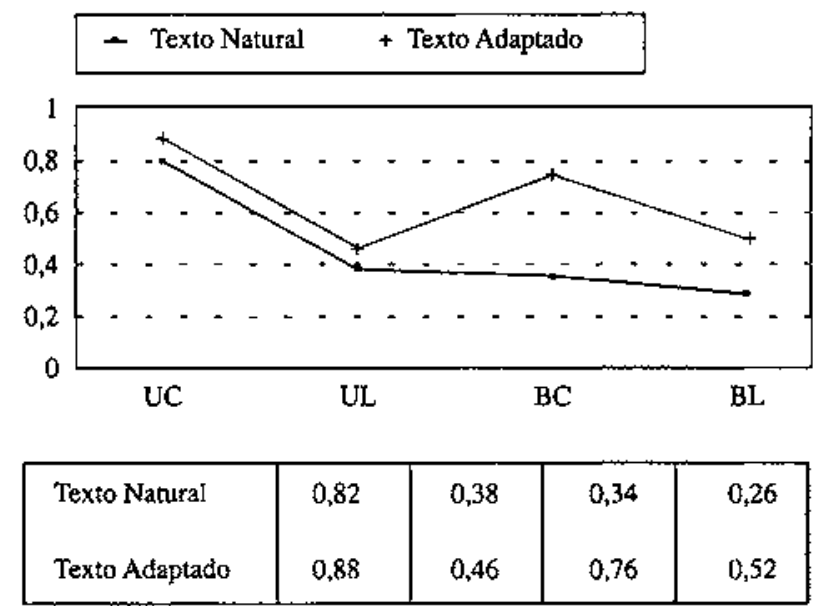

$\mathrm{p}<.001$, texto, $\mathrm{F}(1,77)=44,6, \mathrm{p}<.001, \mathrm{y}$ la interaccion entre las dos primeras variables, $F(1,77)=18,6, p<.001$.

-Prueba de captación de ideas principales: estrategias de lectura, $F(1,77)=4.0, p<.05, y$ texto, $F(1,77)=22.3$, $\mathrm{p}<.001$.

-Prueba de comprensión: estrategias de lectura, $\mathrm{F}(1,77)$ $=8,5, \mathrm{p}<.01$, conocimiento previo, $\mathrm{F}(1,77)=26,8$, $\mathrm{p}<.001$, texto, $F(1,77)=13,5, p<.001$, interacción entre las dos primeras, $\mathrm{F}(1,77)=5,6, \mathrm{p}<.05$, y la primera y la tercera, $F(1,77)=5,8, p<.05$.

En la prueba de conocimiento previo vemos que los sujetos de ciencias tienen un conocimiento previo significativamente más alto que los de letras $y$, por tanto, la identificación efectuada de la variable conocimiento previo con la especialidad cursada, ciencias o letras, tiene validez estadística. Además el texto no produjo diferencias significativas, lo que implica una distribución de sujetos acorde con nuestras premisas.

En las tres restantes pruebas se observan niveles de significación siempre menores de .05 para las variables texto y estrategias de lectura, junto con una notable mejora en los bachilleres que leyeron el texto adaptado. Dicha mejora llega a ser significativa en recuerdo y en comprensión: la prueba t post-hoc dio como resultado $\mathrm{p}<05$ entre las parejas BCN/BCA y BLN/BLA.

\section{CONCLUSIONES}

El texto adaptado produjo una mejora global y significativa en todas las pruebas realizadas (Vidal-A barca et 
al. 1991). Así pues, y dentro de los límites de nuestra investigación, se confirma nuestra hipótesis y se constata el papel crucial de las características textuales de los textos de ciencias en tareas usuales de aprendizaje académico.

También, colateralmente, se ratifica la idoneidad de la manipulación de variables textuales llevada a cabo en el texto adaptado. Ello comporta que en pos de la consectición de textos instruccionales efectivos en ciencias, y sobre todo para bachilleres, no se puede soslayar el conjunto de variables textuales incluido en este estudio y que hemos agrupado en: contenido, estructura organizativa, cohesión y estructura superficial.

\section{REFERENCIAS BIBLIOGRÁFICAS}

AARONSON, D. y FERRES, S., 1984. The word-by-word reading paradigm: an experimental and theorical approach, en D.E. Kieras, M.A. Just, New Methods in reading comprehension research. (Erlbaum: Hillsdale).

AGUIRRE DE CARCER, 1., 1983. Dificultades en la comprension de las explicaciones de los libros de texto de física, Enseñanza de las Ciencias, 1, pp. 92-98.

ANDERSON, T.H. y ARMBRUSTER, B.B.,1984.Content area textbooks, en R.C. Anderson, J. Osborne y R.T. Tierney (eds.), Learning to read in American schools, (Erlbaum: Hillsdale)

ANDERSON T.H. y ARMBRUSTER, B.B., 1986. Readable textbook, or selecting a textbook is not like buying a pair of schoes, en J. Orasau (ed.), Reading Comprehension: from research to practice. (Erlbatum: Hillsdale).

ANDERSON, J.R. y BOWER, G.H., 1973. Human associative memory. (John Wiley: New York).

ANDERSON, R.C. y PEARSON, D.P., 1984, A schema theoretic view of basic processes in reading comprehension, en D.P. Pearson (ed.), Handbook of reading research. (Longman: New York).

ANDERSSON, B.,1990. Pupils' conceptions of matter and its transformations, Studies in Science Education, 18, pp. 53-85.

ASTOLFI, J.P.,1988. El aprendizaje de conceptos científicos: aspectosepistemológicos, cognitivos y lingúísticos, Enseñanza de las Ciencias, 6, pp. 147-155.
No obstante, resta por discernir el efecto individual o por grupos de todas estas variables, y éste es precisamente uno de los problemas que queda abierto. El otro es investigar el efecto de actividades y figuras en el texto. Ambos serán objeto de investigación en un futuro inmediato.

Por otro lado, se ha comprobado la importancia de las estrategias lectoras en los procesos imbricados en la construcción de significado a partir de un texto, de aquí se infiere la necesidad de programas de instrucción específicos para mejorar las estrategias de lectura de nuestros bachilleres si queremos que la interacción entre lector y texto conduzca a un mayor aprendizaje.

AUSUBEL, D., 1976, Psicología educativa. Un punto de vista cognoscitivo. (Trillas: México).

BEAN, T., SINGER, H. y CONAN, S., 1985. Analogical study guides: Improving comprehension in Sciences, Journal of Reading, 29, pp. 246-250.

BERNÁRDEZ, E., 1982. Introducción a la lingüística del texto. (Espasa-Calpe: Madrid).

]3RITTON, B.K., GLYNN, S.M., MEYER, B.J.F. y PENLAND, M.J., 1982. Effects of text structure on use of cognitive capacity during reading, Journal of Educational Psychology, 74, pp. 51-61.

BRITTON, B.K., VAN DUSEN, L., GULGÖZ, S. y GLYNN, S.M., 1989. Instructional texts rewritten by five experts teams: revisions and retention improvements, Journal of Educational Psychology, 81, pp. 226-239.

BROOKS, L.W. y DANSEREAU, D.F., 1983. Effects of structural schema training and text organization on expository prose processing, Journal of Educational Psychology, 75, pp.811820 .

BROWN, A.L., DAY, J.D. y IONES, R.S., 1983. The development of plans for summarizing texts, Child Development, $54, \mathrm{pp}$. 968.979.

BUNGE, M., 1982. Teoria y realidad. (Ariel: Barcelona).

BUNGE, M., 1985.Lainvestigacióncientifica. (Ariel: Barcelona).

CANDEL, A., SATOCA, I., SOLER, J.B. y TENT, J.J., 1988. Física y Química de Segundo de BUP. (Anaya: Madrid).

CAREY, S., 1986. Cognitive Science and Science Education, American Psychologist, 41, pp. 1123-1130. 
CORRAL IÑIGO, A., 1987. Consideraciones acerca de la realización de textos didácticos para la enseñanza a distancia. (UNED: Madrid).

CRAIK, F.I.M. y LOCKHART, R.S., 1972. Levels of processing: a framework for memory research, Journal of Verbal Learning and Verbal Behavior, 11, pp. 671-684.

DANNER, F.W., 1976. Children's understanding of intersentence organization in the recall of short descriptive passages, Journal of Educational Psychology, 68, pp. 174-183.

DEE LUCAS, D. y LARKIN, J.H., 1990. Organization and comprehensibility in scientific proofs, or «Consider a particle p...", Journal of Educational Psychology, 82, pp. 701-714.

DRIVER, R., 1988. Un enfoque constructivista parael desarrollo del currículo en ciencias, Enseñanza de las Ciencias, $6, \mathrm{pp}$. 109-120.

DRIVER, R., 1989. Students' conceptions and the learning of science, International Journal of Sctence Education, $1 \mathrm{I}, \mathrm{pp}$. $481-490$.

DRIVER, R., GUESNE, E. y TIBERGUIEN, A., 1989. Ideas científicas en la infancia y en la adolescencia. (MEC y Morata: Madrid).

DUFFY, J.M., HIGGINS, L., MEHLEMBACHER, B., COCHRAM, C., WALLACE, D., HILL, C., HAUGEN, D., MCCAFFREY, M., BUENET, R., SLOANE,S. y SMITH, S., 1989. Models for design of instructional text, Reading research quartely, 24, pp. 434-456.

DUPIN, J.J. y IOSHUA, S., 1989. Analogies and «modeling Analogies» in teaching: Some examples in basic electricity, Science Education, 73, pp. 207-224.

DUPIN, J.J. y JOSHUA, S., 1990. Una analogía térmica para la enseñanza de la corriente continua en electricidad: đescripción y evaluación, Enseñanza de las Ciencias, 8, pp. 119-126.

FERGUSON-HESSLER, M.G. y DE JONG, T., 1990. Studying Physics Texts: Differences in study processes between good and poor performers, Cognition and Instruction, 7, pp. 4154.

GARCÍA MADRUGA, J.A. Y MARTÍN CORDERO, J.I., 1987. Aprendizaje, comprensión y retención de textos. (UNED: Madrid).

GLLBERT, S.W., 1989. An evaluation of the use of analogy, simite, and metaphor in science texts, Journal of Research in Science Teaching, 26, pp. 315-327.

GILBERT, S.W., 1991. Model building and a definition of science, Journal of research in Science Teaching, 28, pp. 73.79.

GOLDEN, J., HASLETT, B. y GAUNTT, H., 1988. Structure and content in Eighth-Graders' summary essays, Discourse processes, II, pp. 139-162.

HEALY, V.C., 1989. The effects of advance organizer and prerequisite knowledge passages on the learning and retention of science concepts, Journal of research in Science Teaching, 26 , pp. $627-642$.

HIERREZUELO, J. y MONTERO, A., 1989. La ciencia de los alumnos. Su utilización en la didáctica de la física y la química. (MEC y Laia: Barcelona).
HODSON, D., 1988. Toward a philosophically more valid science currículum, Science Education, 72, pp. 19-40.

HYND, C. y ALVERMANN, D., 1986. The role of refutation text in overcoming difficulty with science concepts, Journal of reading, 29 , pp. 440-447.

KIERAS.D.E., 1978.Good and bad structure in simple paragraphs; effects on apparent theme, reading them and recall, Journal of Verb al Learning and Verbal Behavior, 17, pp. 13-28.

KIERAS, D.E., 1985. Thematic processes in the comprehension of technical prose, en B. K. Britton y J. B. Black (eds.), Understanding expository text. (ErIbautm: Hillsdale).

KINTSCH, W., 1972, Notes on the structure of semantic memory, en E. Tluving, W. Donaldson (eds.), Organization of memory. (Academic Press: Nueva York).

KINTSCH, W., 1974. The representation of meaning in memory. (Erlbatum: Hillsdale).

KINTSCH, W. y VAN DIJK, T.A., 1978. Toward a model of discourse comprehension and production, Psychological review, 85, pp. 363-394.

KINTSCH, W, y YARBROUGH, J.C., 1982. Role of rethorical structure in prose comprehension, Journal of Educational Psychological, 74, pp. 824-834.

KUHN, T.S., 1987. La tensión esencial. Estudios selectos sobre la tradición y cambio en el ámbito de la ciencia. (FCE: México).

LEONARD, W.H., 1987. Does the presentation style of questions inserted into text infuence understanding and retention of science concepts?,Journal of Research in Science Teaching, 24 , pp. 27-37.

LEONARD W.H. y LOWERY, L.F., 1984. The effects of question types in textual reading upon retention of biology concepts, Journal of research in science teaching, $21, \mathrm{pp}$. 377-384.

LLORENS, J.A., 1988. La concepción corpuscular de lá materia. Obstáculos epistemológicos y problemas de aprendizaje, Investigación en la escuela, 4, pp. 33-48.

LLORENS, J.A., DE JAIME, M.C. y LLOPIS, R., 1989. La función del lenguaje en un enfoque constructivista del aprendizaje de las ciencias, Enseñanza de las Ciencias, 7,pp. 111-119.

MARTINAND, J.L., 1986. Enseñanza y aprendizaje de la modelización, Enseñanza de las Ciencias, 4, pp. 45-50.

MAYER, R.E., 1985. Structural analysis of science prose: Can we increase problem-solving perfomance?, en B.K.Britton y J.B. Black (eds.), Understanding Expository texts. (Erlbaum: Hillsdale).

MAYER, R.E., 1989. Systematic thinking fostered by illustrations in scientific text, Journal of Educational Psychology, 81, pp. 240-246.

MAYER, R.E. y GALLINI, J.K., 1990. When is an illustration worth ten thousand words?, Journal of Educational Psychology, 82 , pp. 715-726.

MEHEUT, M., LARCHER, C. y CHOMAT, A, 1988. Modelos de particulas en la iniciación a las ciencias físicas, Enseñanza de las Ciencias, 6, pp. 231-238. 
MERRILL, M.D., 1983. Component Display Theory, en Ch. M. Reigeluth (ed.), Instructional Design Theories and Models: an overview of their current status. (Erlbaum: Hillsdale).

MERRILL, M.D., 1987. A lesson based on the component display theory, en Ch.M. Reigeluth (ed.), Instructional theories in action. Lessons Illustrating selected theories and models. (Erlbaum: Hillsdale).

MEYER, B.J.F., 1984. Textdimensions and cognitive processing, en H. MandI. N. Stein, T. Trabasso (eds.), Learning from texts. (Hillsdale: Erlbaum).

MEYER, L.A., CRUMMEY,L. y GREER,E.A., 1988.Elementary science tetxbooks: Their contents, text characteristics, and comprehensibility, Journal of Research in Science Teaching, 25 , pp. $435-463$

OTERO, J.C., 1985. Assimilation problems in traditional representations of scientific knowledge, European Journal of Science Education, 7, pp. 361-369.

OTERO, J.C., 1989. La producción y la comprensión de la ciencia: la elaboracion en el aprendizaje de la ciencia escolar, Enseñanza de las Ciencias, 7, pp. 223-228.

OTERO, J.C., 1990. Variables cognitivas y metacognitivas en la comprensión de textos científicos: el papel de los esquemas y el control de la propia comprensión, Enseñanza de las Ciencias, 8, 17-22.

OTERO, J.C. y CAMPANARIO, J.M., 1990. Comprehension evaluation and regulation in learning from science texts, Journal of Research in Science Teaching, 27, pp. 447-460.

PEDERSEN, J, BONNTETTRER, R.J., CORKILL, A.J. y GLOVER, J.A., 1988. Learning Chemistry from text: The effect of decision making, Journal of Research in Science Teaching, 25, pp. 15-21.

PERFETTI, Ch. A., 1986. Capactdad de lectura, en R.J. Sternberg (ed.): Las capacidades humanas. Un enfoque desde el procesamiento de la información. (Labor: Barcelona).

REIGELUTH, Ch. M., I987, Lesson Blueprints based on the Elaboration Theory of instruction, en Ch. M. Reigeluth (ed.), Instructional theories in action: Lessons illustrating selected theories and models. (Erlbaum: Hillsdale).

REIGELUTH, Ch.M. y STEIN, F.S., 1983. The Elaboration Theory of instruction, en Ch. M. Reigeluth (ed.), Instructional Design Theories and Models: an overview of their current status. (Erlbaum: Hillscale).

RENNER,J.W.,ABRAHAM, M.R.,BROSS, E. y MAREK, E.A., 1990. Understandings and misunderstandings of eighth graders of four physics concepts found in textbooks, Journal of Research in Science Teaching, 27, pp. 35-54.

RENSTRÖM, L., ANDERSSON, B. y MARTON, T., 1990. Students' conceptions of matter, Journal of Educational Psychology, 82, pp. 555-569.

REYNOLDS, R.E. y BAKER, D.R., 1987. The utility of graphical representations in text: Some theoretical and empirical issues, Journal of Research in Science Teaching, 24, pp. 161-173.

ROSSI, J.P., 1990. The function of frame in the comprehension of scientific text, Journal of Educational Psychology, 82, pp. $727-732$.
ROTH, K. y ANDERSON, Ch., 1988. Promoting conceptual change learning from science textbooks, en P. Ramsden (ed.), Improving learning: New perspectives. (Kogan: Londres).

RUMELHART, D.E., LINDSAY, P.H. y NORMAN, D.A., 1972 , A process model for long-term memory, en $E$. Tulving, $W$ Donaldson (eds.), Organization and memory. (Academic Press: NuevaYork).

SANTELICES, L., 1990. La comprensión de lectura en textos de ciencias, Enseñanza de las Ciencias, 8, pp. 59-64.

SARI, I.F. y REIGELUTH, Ch.M., 1982. Writing and Evaluating textbooks: Contributions from instructional theory, en D.H. Jonassen (ed.): The technology of text. (Greenboro Educational technology publications: Carolina).

SCARDAMALIA, M. y BEREITER, C., 1984. Development of strategies in text processing, en H. Mandl, N.L. Stein y T. Trabasso (eds.), Learning and Comprehension Text. (Erlbaum: Hillsdale).

SOLAZ PORTOLÉS, J.J., 1992. El papel del texto en didáctica de las ciencias: una aproximación empírica a la comprensión lectora. Tesis de tercer ciclo no publicada. Universitat de València.

SPIRO, R.J., 1980. Constructive processes in prosecomprehension and recall, en R.J. Spiro, B.C. Bruce y W.F. Brewer (eds.). Theorical issues in reading comprehension. (Erlbaum: Hillsdale).

STAVER, J.R. y BAY, M., 1989. Analysis of the conceptual structure and reasoning demands of elementary science texts at the primary (K-3) level, Journal of Research in Science Teaching, 26, pp. 329-349.

STINNER, A., 1989. The teaching of Physics and the contexts of inquiry: From Aristotle to Einstein, Science Education, 73, pp. 59I-605.

STOREY VASU, E. y HOWE, A. C., 1989. The effect of visual and verbal modes of presentation on children's retention of images and words, Journal of Research in Science Teaching, 26, pp. 401-407.

STRUBE, P., 1989a. The notion of style in physics textbooks, Journal of Research in Science Teaching, 26, pp. 291-299.

STRUBE, P., 1989b. A content analysis of arguments and explications presented to students in physical science textbooks: a model and a example, International Journal of Science Education, 1I, pp. 195-202.

V'AN DIJK, J.A. y KINTSCH, W., 1983. Strategies of discourse comprehension. (Academic Press: New York).

VIDAL-ABARCA, E., 1989. La comprensión lectora de ideas principales en textos expositivos del ciclo medio de la EGB: Programa de instrucción y procesos explicativos. Tesis doctoral no publicada. Universitat de Valencia.

VIDAL-ABARCA, E., SANJOSÉ, V. y SOLAZ PORTOLÉS, J.J. 1991. Una aproximación empírica a la comprensión lectora en ciencias. El papel preponderante del texto. Actas del I Congreso Internacional de Psicología y Educación, p. 206. Madrid, 21-23 de noviembre.

WANDERSEE, J.H., 1988. Ways students read texts, Journal of Research in Science Teaching, 25, pp. 69-84. 
WILLIAMS, R.L. y YORE,L.D., 1985. Content, format, gender, and grade level differences in elementary students' ability to read science materials as measured by the cloze procedure, Journal of Research in Science Teaching, 22, pp. 81-88.

WILSON, P.T. y ANDERSON, R.C., 1986. What they don't know will hurt them: The role of prior knowledge in comprehension, en J. Orasam (ed.), Reading comprehension: from research to practice (Erlbaum: Hillsdale).

WOOD, T.L. y WOOD, W.L., Assessing potential difficulties in comprehending fourth grade science textbooks, Science Education, 72, pp. 561-574. 